\title{
A LIMIT THEOREM FOR A WEISS EPIDEMIC PROCESS
}

\author{
A. V. KALINKIN ${ }^{* * *}$ AND \\ A. V. MASTIKHIN, ${ }^{* * *}$ Bauman Moscow State Technical University
}

\begin{abstract}
For a Markov two-dimensional death-process of a special class we consider the use of Fourier methods to obtain an exact solution of the Kolmogorov equations for the exponential (double) generating function of the transition probabilities. Using special functions, we obtain an integral representation for the generating function of the transition probabilities. We state the expression of the expectation and variance of the stochastic process and establish a limit theorem.
\end{abstract}

Keywords: Markov epidemic process; transition probabilities; exponential generating function; closed solution; branching property

2010 Mathematics Subject Classification: Primary 60F99; 60J27; 60K35

Secondary 60J80; 92D30

\section{The Markov epidemic process}

We consider a time-homogeneous Markov process

$$
\xi(t)=\left(\xi_{1}(t), \xi_{2}(t)\right), \quad t \in[0, \infty),
$$

on the set of states

$$
N^{2}=\left\{\left(\alpha_{1}, \alpha_{2}\right), \alpha_{1}, \alpha_{2}=0,1, \ldots\right\}
$$

with transition probabilities

$$
P_{\left(\beta_{1}, \beta_{2}\right)}^{\left(\alpha_{1}, \alpha_{2}\right)}(t)=\mathbb{P}\left\{\xi(t)=\left(\beta_{1}, \beta_{2}\right) \mid \xi(0)=\left(\alpha_{1}, \alpha_{2}\right)\right\} .
$$

Let us suppose that the transition probabilities have the following form as $t \rightarrow 0+, \mu \geq 0$ (see [3] and [15]),

$$
\begin{gathered}
P_{\left(\alpha_{1}, \alpha_{2}-1\right)}^{\left(\alpha_{1}, \alpha_{2}\right)}(t)=p_{1} \alpha_{1} \alpha_{2} t+o(t), \quad P_{\left(\alpha_{1}+1, \alpha_{2}-1\right)}^{\left(\alpha_{1}, \alpha_{2}\right)}(t)=p_{2} \alpha_{1} \alpha_{2} t+o(t), \\
P_{\left(\alpha_{1}-1, \alpha_{2}\right)}^{\left(\alpha_{1}, \alpha_{2}\right)}(t)=\mu \alpha_{1} t+o(t), \quad P_{\left(\alpha_{1}, \alpha_{2}\right)}^{\left(\alpha_{1}, \alpha_{2}\right)}(t)=1-\left(\alpha_{1} \alpha_{2}+\mu \alpha_{1}\right) t+o(t),
\end{gathered}
$$

where $p_{1} \geq 0, p_{2} \geq 0, p_{1}+p_{2}=1$.

Let us introduce the generating functions of the transition probabilities $\left(\left|s_{1}\right| \leq 1,\left|s_{2}\right| \leq 1\right)$,

$$
F_{\left(\alpha_{1}, \alpha_{2}\right)}\left(t ; s_{1}, s_{2}\right)=\sum_{\beta_{1}, \beta_{2}=0}^{\infty} P_{\left(\beta_{1}, \beta_{2}\right)}^{\left(\alpha_{1}, \alpha_{2}\right)}(t) s_{1}^{\beta_{1}} s_{2}^{\beta_{2}}, \quad\left(\alpha_{1}, \alpha_{2}\right) \in N^{2} .
$$

Received 23 November 2012; revision received 12 March 2014.

* Postal address: Department of Higher Mathematics, Bauman Moscow State Technical University, 2nd Bauman St., 5 , 105005 Moscow, Russia.

** Email address: kalinkin@bmstu.ru

*** Email address: mastikhin@yandex.ru 
The second (forward) system of the Kolmogorov differential equations for the transition probabilities of the process $\xi(t)$ is equivalent to the partial differential equation (see [3], [5], [6]),

$$
\frac{\partial F_{\left(\alpha_{1}, \alpha_{2}\right)}}{\partial t}=\left(p_{2} s_{1}^{2}+p_{1} s_{1}-s_{1} s_{2}\right) \frac{\partial^{2} F_{\left(\alpha_{1}, \alpha_{2}\right)}}{\partial s_{1} \partial s_{2}}+\mu\left(1-s_{1}\right) \frac{\partial F_{\left(\alpha_{1}, \alpha_{2}\right)}}{\partial s_{1}},
$$

with the initial condition $F_{\left(\alpha_{1}, \alpha_{2}\right)}\left(0 ; s_{1}, s_{2}\right)=s_{1}^{\alpha_{1}} s_{2}^{\alpha_{2}}$.

An event $\left\{\xi(t)=\left(\alpha_{1}, \alpha_{2}\right)\right\}$ is interpreted as the existence of a population of $\alpha_{1}$ particles of type $T_{1}$ and $\alpha_{2}$ particles of type $T_{2}$ at time $t$. The following description is customary in probabilistic models of the spreading of an epidemic [2]. The particles of type $T_{1}$ are interpreted as sick individuals and the particles of type $T_{2}$ as healthy individuals susceptible to the infectious disease. We can assume that after a random time interval $\tau_{\left(\alpha_{1}, \alpha_{2}\right)}^{2}, \mathbb{P}\left\{\tau_{\left(\alpha_{1}, \alpha_{2}\right)}^{2} \leq t\right\}=1-\mathrm{e}^{-\alpha_{1} \alpha_{2} t}$, contact is made between a particle of type $T_{1}$ and a particle of type $T_{2}$. This pair of particles is transformed into a particle of type $T_{1}$ with the probability $p_{1}$ (the infected individual is removed from the population) - the process goes to the state $\left(\alpha_{1}, \alpha_{2}-1\right)$, or is transformed into a pair of particles of type $T_{1}$ with the probability $p_{2}$ (the infected individual is not removed from the population $)$ - the process goes to the state $\left(\alpha_{1}+1, \alpha_{2}-1\right)$. Besides, after a random time interval $\tau_{\left(\alpha_{1}, \alpha_{2}\right)}^{1}, \mathbb{P}\left\{\tau_{\left(\alpha_{1}, \alpha_{2}\right)}^{1} \leq t\right\}=1-\mathrm{e}^{-\mu \alpha_{1} t}$, one of the particles of type $T_{1}$ dies - the process goes to the state $\left(\alpha_{1}-1, \alpha_{2}\right)$. The random variables $\tau_{\left(\alpha_{1}, \alpha_{2}\right)}^{1}$ and $\tau_{\left(\alpha_{1}, \alpha_{2}\right)}^{2}$ are independent and the process $\xi(t)$ remains in the state $\left(\alpha_{1}, \alpha_{2}\right)$ for a random time $\tau_{\left(\alpha_{1}, \alpha_{2}\right)}=\min \left(\tau_{\left(\alpha_{1}, \alpha_{2}\right)}^{1}, \tau_{\left(\alpha_{1}, \alpha_{2}\right)}^{2}\right)$. An example of a realization of the process is shown in Figure 1, case (a).

The process $\xi(t)$ is called a Weiss epidemic process [15] in the $p_{1}=1$ case and a BartlettMcKendrick epidemic process [3] in the $p_{2}=1$ case. The process $\xi(t)$ belongs to a special class of Markov processes introduced by Sevast'yanov [13]. The generalization of such a process with two kinds of particles $T_{1}, T_{2}$ and two complexes of interaction is described in [7]. An equation of this type is also the generalization of (3).

There are numerous publications devoted to explicit solutions of equations for different Markov epidemic processes (see, for example, [2], [5], [7], the survey [8], [9]-[11], [14], and [15]). For (3) in the $p_{1}=1$ case the expression for the transition probabilities is well known [15] (see Remark 1 below). In the $p_{2}=1$ case, Gani [5] found the solution of (3) by the Laplace transform method and wrote out the transition probabilities. Siskind [14]

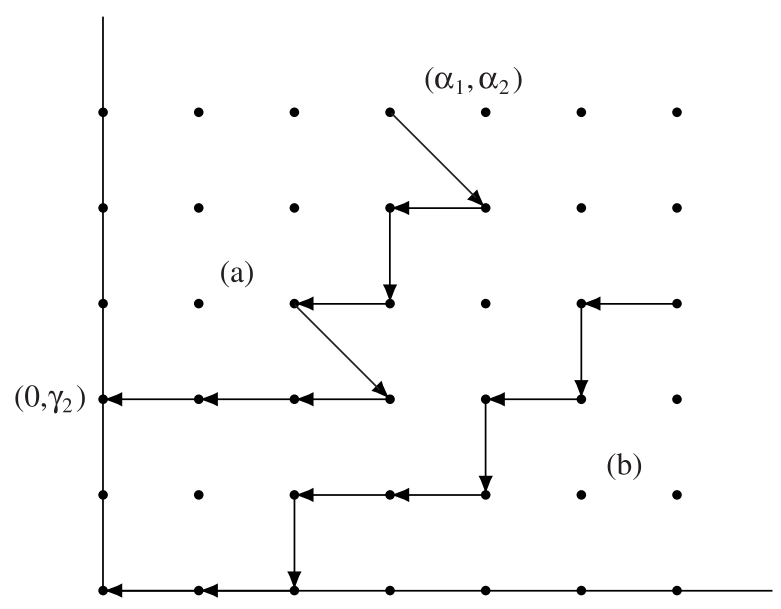

FIGURE 1: Jumps for a Markov process. 
solved (3) by direct recurrent integration and obtained the same equations. A few simpler series for the solution of (3) were obtained by Sakino [11]. However, all these expressions for $F_{\left(\alpha_{1}, \alpha_{2}\right)}\left(t ; s_{1}, s_{2}\right)$ have very complicated forms; for instance, the solution obtained in [5] fills two journal pages. These equations are of little use in the study of asymptotic properties of the random process $\xi(t)$.

In this paper the construction of the solution for (3) is based on the statements of the theory of branching processes with independent particles [1], [12].

\section{The nonlinear branching property and the solving of problem (3)}

Let us consider the quite simple branching process which is the Markov process on the set of states $N^{2}$ such that the transition probabilities have the following form as $t \rightarrow 0+, \mu_{1} \geq 0$, $\mu_{2} \geq 0$

$$
\begin{gathered}
P_{\left(\alpha_{1}-1, \alpha_{2}\right)}^{\left(\alpha_{1}, \alpha_{2}\right)}(t)=\mu_{1} \alpha_{1} t+o(t), \quad P_{\left(\alpha_{1}, \alpha_{2}-1\right)}^{\left(\alpha_{1}, \alpha_{2}\right)}(t)=\mu_{2} \alpha_{2} t+o(t), \\
P_{\left(\alpha_{1}, \alpha_{2}\right)}^{\left(\alpha_{1}, \alpha_{2}\right)}(t)=1-\left(\mu_{1} \alpha_{1}+\mu_{2} \alpha_{2}\right) t+o(t) .
\end{gathered}
$$

For the generating function of the transition probabilities (2) the partial differential equation holds (see [1], [12, Section 4.3, Equation (12)]) such that

$$
\frac{\partial F_{\left(\alpha_{1}, \alpha_{2}\right)}}{\partial t}=\mu_{1}\left(1-s_{1}\right) \frac{\partial F_{\left(\alpha_{1}, \alpha_{2}\right)}}{\partial s_{1}}+\mu_{2}\left(1-s_{2}\right) \frac{\partial F_{\left(\alpha_{1}, \alpha_{2}\right)}}{\partial s_{2}},
$$

with initial conditions $F_{\left(\alpha_{1}, \alpha_{2}\right)}\left(0 ; s_{1}, s_{2}\right)=s_{1}^{\alpha_{1}} s_{2}^{\alpha_{2}}$.

The state $\left(\alpha_{1}, \alpha_{2}\right)$ of the process is interpreted as the existence of $\alpha_{1}$ particles of type $T_{1}$ and $\alpha_{2}$ particles of type $T_{2}$. At a random time $\tau_{\left(\alpha_{1}, \alpha_{2}\right)}^{1}, \mathbb{P}\left\{\tau_{\left(\alpha_{1}, \alpha_{2}\right)}^{1} \leq t\right\}=1-\mathrm{e}^{-\mu_{1} \alpha_{1} t}$, one of the particles of type $T_{1}$ dies and the process passes to the state $\left(\alpha_{1}-1, \alpha_{2}\right)$. Besides, after a random time $\tau_{\left(\alpha_{1}, \alpha_{2}\right)}^{2}, \mathbb{P}\left\{\tau_{\left(\alpha_{1}, \alpha_{2}\right)}^{2} \leq t\right\}=1-\mathrm{e}^{-\mu_{2} \alpha_{2} t}$, one of the particles of type $T_{2}$ dies and the process passes to the state $\left(\alpha_{1}, \alpha_{2}-1\right)$. The random variables $\tau_{\left(\alpha_{1}, \alpha_{2}\right)}^{1}$ and $\tau_{\left(\alpha_{1}, \alpha_{2}\right)}^{2}$ are independent and the process remains in the state $\left(\alpha_{1}, \alpha_{2}\right)$ during the random time $\tau_{\left(\alpha_{1}, \alpha_{2}\right)}=\min \left(\tau_{\left(\alpha_{1}, \alpha_{2}\right)}^{1}, \tau_{\left(\alpha_{1}, \alpha_{2}\right)}^{2}\right)$. The example of a realization of the death-process is shown in Figure 1, case (b).

The solution of the first order equation (4) is found by standard methods and it has the branching property ([12, Section 4.2, Equation (3)])

$$
F_{\left(\alpha_{1}, \alpha_{2}\right)}\left(t ; s_{1}, s_{2}\right)=\left(1-\mathrm{e}^{-\mu_{1} t}+s_{1} \mathrm{e}^{-\mu_{1} t}\right)^{\alpha_{1}}\left(1-\mathrm{e}^{-\mu_{2} t}+s_{2} \mathrm{e}^{-\mu_{2} t}\right)^{\alpha_{2}} .
$$

In this paper Theorem 1 establishes the solution of the second order partial differential equation (3) for the case of the Weiss epidemic process. This solution gives us the generalization of (5). We use the method of [8] to obtain such a representation for $F_{\left(\alpha_{1}, \alpha_{2}\right)}\left(t ; s_{1}, s_{2}\right)$. We consider both the first and the second Kolmogorov equations for the exponential (double) generating function of the transition probabilities. The solution of the system is obtained in the form of a series with separating variables. Then we construct an integral representation for the series which contains special functions. Further manipulation with the explicit solution provides the form that is analogous to the nonlinear property (5).

Equation (3) in Theorem 1 is solved when $p_{1}=1$, however, the proposed method is applicable even if $p_{1}<1$ despite the difficulties of the transformation technique. 


\section{The closed solution of the Kolmogorov equations}

Let us consider the process $\xi(t)$ when $p_{1}=1$. We introduce an exponential generating function (see [8] and [13])

$$
\begin{aligned}
\mathcal{F}\left(t ; z_{1}, z_{2} ; s_{1}, s_{2}\right) & =\sum_{\alpha_{1}, \alpha_{2}=0}^{\infty} \frac{z_{1}^{\alpha_{1}} z_{2}^{\alpha_{2}}}{\alpha_{1} ! \alpha_{2} !} F_{\left(\alpha_{1}, \alpha_{2}\right)}\left(t ; s_{1}, s_{2}\right) \\
& =\sum_{\alpha_{1}, \alpha_{2}, \beta_{1}, \beta_{2}=0}^{\infty} \frac{z_{1}^{\alpha_{1}} z_{2}^{\alpha_{2}}}{\alpha_{1} ! \alpha_{2} !} P_{\left(\beta_{1}, \beta_{2}\right)}^{\left(\alpha_{1}, \alpha_{2}\right)}(t) s_{1}^{\beta_{1}} s_{2}^{\beta_{2}} .
\end{aligned}
$$

For the process under consideration, we can write down the first (backward) and the second (forward) systems of the Kolmogorov differential equations for the transition probabilities $P_{\left(\beta_{1}, \beta_{2}\right)}^{\left(\alpha_{1}, \alpha_{2}\right)}(t)$ in the form of two equations with partial derivatives of the second order [8], such that

$$
\begin{gathered}
\frac{\partial \mathcal{F}}{\partial t}=z_{1} z_{2}\left(\frac{\partial \mathcal{F}}{\partial z_{1}}-\frac{\partial^{2} \mathcal{F}}{\partial z_{1} \partial z_{2}}\right)+\mu z_{1}\left(\mathscr{F}-\frac{\partial \mathcal{F}}{\partial z_{1}}\right), \\
\frac{\partial \mathcal{F}}{\partial t}=\left(s_{1}-s_{1} s_{2}\right) \frac{\partial^{2} \mathcal{F}}{\partial s_{1} \partial s_{2}}+\mu\left(1-s_{1}\right) \frac{\partial \mathcal{F}}{\partial s_{1}},
\end{gathered}
$$

with the initial condition $\mathcal{F}\left(0 ; z_{1}, z_{2} ; s_{1}, s_{2}\right)=\mathrm{e}^{z_{1} s_{1}+z_{2} s_{2}}$.

Furthermore, we need the function, $x>0, y>0$,

$$
H(x, y)=\int_{0}^{\infty} \int_{0}^{\infty} J_{0}(2 \sqrt{u x}) J_{0}(2 \sqrt{v y})_{0} F_{2}(1,1 ;-u v) \mathrm{d} u \mathrm{~d} v
$$

where $J_{0}(z)$ is the Bessel function of order zero and ${ }_{0} F_{2}(1,1 ; z)$ is the generalized hypergeometric function,

$$
J_{0}(z)=\sum_{k=0}^{\infty} \frac{(-1)^{k}(z / 2)^{2 k}}{k ! k !}, \quad{ }_{0} F_{2}(1,1 ; z)=\sum_{k=0}^{\infty} \frac{z^{k}}{(k !)^{3}} .
$$

Theorem 1. For the Markov process $\xi(t)$ on the set of states $N^{2}$ under condition (1) and $p_{1}=1$, the generating function of the transition probabilities is as follows. Let

$$
\begin{aligned}
& F_{\left(\alpha_{1}, \alpha_{2}\right)}\left(t ; s_{1}, s_{2}\right) \\
& =\int_{0}^{\infty} \int_{0}^{\infty}\left(\left(s_{1} \mathrm{e}^{-(x+\mu) t}\right)^{\alpha_{1}}\left(1-\mathrm{e}^{-y}+s_{2} \mathrm{e}^{-y}\right)^{\alpha_{2}}\right. \\
& \left.\quad+\int_{0}^{\infty}\left(\frac{1}{2 \pi \mathrm{i}} \oint_{0+} \varphi_{1}^{\alpha_{1}}\left(t ; x, u ; s_{1}\right) \varphi_{2}^{\alpha_{2}}\left(y, v ; s_{2}\right) \mathrm{e}^{(u-\mu) v} \mathrm{~d} u\right) \mathrm{d} v\right) H(x, y) \mathrm{d} x \mathrm{~d} y,
\end{aligned}
$$

where the functions $\varphi_{1}\left(t ; x, u ; s_{1}\right)$ and $\varphi_{2}\left(y, v ; s_{2}\right)$ are linear with respect to variables $s_{1}$ and $s_{2}$, such that

$$
\varphi_{1}\left(t ; x, u ; s_{1}\right)=\mu \frac{1-\mathrm{e}^{-(x+\mu) t}}{u}+s_{1} \mathrm{e}^{-(x+\mu) t}, \quad \varphi_{2}\left(y, v ; s_{2}\right)=1-\mathrm{e}^{-y-v}+s_{2} \mathrm{e}^{-y-v} .
$$


Proof. (a) Separating of variables. At the first stage we apply a Fourier method of separating the variables to the system of linear equations (7) and (8). We look for the solution in a form of the series

$$
\mathcal{F}\left(t ; z_{1}, z_{2} ; s_{1}, s_{2}\right)=\sum_{\alpha_{1}, \alpha_{2}=0}^{\infty} A_{\alpha_{1} \alpha_{2}} \widetilde{C}_{\alpha_{1} \alpha_{2}}\left(z_{1}, z_{2}\right) C_{\alpha_{1} \alpha_{2}}\left(s_{1}, s_{2}\right) \mathrm{e}^{-\lambda_{\alpha_{1} \alpha_{2}} t} .
$$

After substitution of (11) into (7) and (8) we obtain the following equations for the functions $\widetilde{C}_{\alpha_{1} \alpha_{2}}\left(z_{1}, z_{2}\right)$ and $C_{\alpha_{1} \alpha_{2}}\left(s_{1}, s_{2}\right)$ :

$$
\begin{gathered}
z_{1} z_{2}\left(\frac{\partial \widetilde{C}_{\alpha_{1} \alpha_{2}}}{\partial z_{1}}-\frac{\partial^{2} \widetilde{C}_{\alpha_{1} \alpha_{2}}}{\partial z_{1} \partial z_{2}}\right)+\mu z_{1}\left(\widetilde{C}_{\alpha_{1} \alpha_{2}}-\frac{\partial \widetilde{C}_{\alpha_{1} \alpha_{2}}}{\partial z_{1}}\right)+\lambda_{\alpha_{1} \alpha_{2}} \widetilde{C}_{\alpha_{1} \alpha_{2}}=0 \\
\left(s_{1}-s_{1} s_{2}\right) \frac{\partial^{2} C_{\alpha_{1} \alpha_{2}}}{\partial s_{1} \partial s_{2}}+\mu\left(1-s_{1}\right) \frac{\partial C_{\alpha_{1} \alpha_{2}}}{\partial s_{1}}+\lambda_{\alpha_{1} \alpha_{2}} C_{\alpha_{1} \alpha_{2}}=0
\end{gathered}
$$

$\alpha_{1}, \alpha_{2}=0,1, \ldots$ According to the conditions for the jumps of the process, (13) has the additional boundary condition ' $C_{\alpha_{1} \alpha_{2}}\left(s_{1}, s_{2}\right)$ is polynomia' (cf. [8, Section 4.2.1]). Then the sequence of 'eigenvalues' $\lambda_{\alpha_{1} \alpha_{2}}=\alpha_{1} \alpha_{2}+\mu \alpha_{1}, \alpha_{1}, \alpha_{2}=0,1, \ldots$, and each $\lambda_{\alpha_{1} \alpha_{2}}$ has the corresponding 'eigenfunction'

$$
C_{\alpha_{1} \alpha_{2}}\left(s_{1}, s_{2}\right)=\left(s_{1}-\frac{\mu}{\alpha_{2}+\mu}\right)^{\alpha_{1}}\left(s_{2}-1\right)^{\alpha_{2}} .
$$

Consequently, (12) has the form

$$
z_{1} z_{2}\left(\frac{\partial \widetilde{C}_{\alpha_{1} \alpha_{2}}}{\partial z_{1}}-\frac{\partial^{2} \widetilde{C}_{\alpha_{1} \alpha_{2}}}{\partial z_{1} \partial z_{2}}\right)+\mu z_{1}\left(\widetilde{C}_{\alpha_{1} \alpha_{2}}-\frac{\partial \widetilde{C}_{\alpha_{1} \alpha_{2}}}{\partial z_{1}}\right)+\left(\alpha_{1} \alpha_{2}+\mu \alpha_{1}\right) \widetilde{C}_{\alpha_{1} \alpha_{2}}=0
$$

According to the conditions for the jumps of the process it follows that we are looking for an analytic solution, for all $z_{1}, z_{2}$,

$$
\widetilde{C}_{\alpha_{1} \alpha_{2}}\left(z_{1}, z_{2}\right)=z_{1}^{\alpha_{1}} z_{2}^{\alpha_{2}} \mathrm{e}^{z_{1} \mu /\left(\alpha_{2}+\mu\right)+z_{2}} .
$$

Therefore, the desired series (11) has the form

$$
\begin{aligned}
& \mathcal{F}\left(t ; z_{1}, z_{2} ; s_{1}, s_{2}\right) \\
& \quad=\sum_{\alpha_{1}, \alpha_{2}=0}^{\infty} A_{\alpha_{1} \alpha_{2}} z_{1}^{\alpha_{1}} z_{2}^{\alpha_{2}} \mathrm{e}^{z_{1} \mu /\left(\alpha_{2}+\mu\right)+z_{2}}\left(s_{1}-\frac{\mu}{\alpha_{2}+\mu}\right)^{\alpha_{1}}\left(s_{2}-1\right)^{\alpha_{2}} \mathrm{e}^{-\left(\alpha_{1} \alpha_{2}+\mu \alpha_{1}\right) t} .
\end{aligned}
$$

Comparing the initial conditions of $\mathcal{F}\left(0 ; z_{1}, z_{2} ; s_{1}, s_{2}\right)=\mathrm{e}^{z_{1} s_{1}+z_{2} s_{2}}$ and the exponent expanding

$$
\begin{aligned}
\mathrm{e}^{z_{1} s_{1}+z_{2} s_{2}} & =\mathrm{e}^{z_{1} s_{1}+z_{2}} \sum_{\alpha_{2}=0}^{\infty} \frac{z_{2}^{\alpha_{2}}}{\alpha_{2} !}\left(s_{2}-1\right)^{\alpha_{2}} \\
& =\mathrm{e}^{z_{2}} \sum_{\alpha_{2}=0}^{\infty} \frac{z_{2}^{\alpha_{2}}}{\alpha_{2} !}\left(s_{2}-1\right)^{\alpha_{2}} \mathrm{e}^{z_{1} \mu /\left(\alpha_{2}+\mu\right)} \mathrm{e}^{z_{1}\left(s_{1}-\mu /\left(\alpha_{2}+\mu\right)\right)} \\
& =\mathrm{e}^{z_{2}} \sum_{\alpha_{2}=0}^{\infty} \frac{z_{2}^{\alpha_{2}}}{\alpha_{2} !}\left(s_{2}-1\right)^{\alpha_{2}} \mathrm{e}^{z_{1} \mu /\left(\alpha_{2}+\mu\right)} \sum_{\alpha_{1}=0}^{\infty} \frac{z_{1}^{\alpha_{1}}}{\alpha_{1} !}\left(s_{1}-\frac{\mu}{\alpha_{2}+\mu}\right)^{\alpha_{1}}
\end{aligned}
$$


we find the values for $A_{\alpha_{1} \alpha_{2}}$. We obtain $A_{\alpha_{1} \alpha_{2}}=1 /\left(\alpha_{1} ! \alpha_{2} !\right)$ and

$$
\begin{aligned}
& \mathscr{F}\left(t ; z_{1}, z_{2} ; s_{1}, s_{2}\right) \\
& \quad=\sum_{\alpha_{1}, \alpha_{2}=0}^{\infty} \frac{z_{1}^{\alpha_{1}} z_{2}^{\alpha_{2}}}{\alpha_{1} ! \alpha_{2} !} \mathrm{e}^{z_{1} \mu /\left(\alpha_{2}+\mu\right)+z_{2}}\left(s_{1}-\frac{\mu}{\alpha_{2}+\mu}\right)^{\alpha_{1}}\left(s_{2}-1\right)^{\alpha_{2}} \mathrm{e}^{-\left(\alpha_{1} \alpha_{2}+\mu \alpha_{1}\right) t} .
\end{aligned}
$$

Absolute convergence of the series (14) for all $z_{1}, z_{2}, s_{1}, s_{2}$ and $t \in[0, \infty)$ is obvious.

(b) The integral representation. We use the following representation of the exponent ([4, Part 2, relation (3.5) and Part 1, Chapter 2, Section 12])

$$
\mathrm{e}^{-\alpha_{1} \alpha_{2} t}=\int_{0}^{\infty} \int_{0}^{\infty} \mathrm{e}^{-\alpha_{1} t x-\alpha_{2} y} H(x, y) \mathrm{d} x \mathrm{~d} y,
$$

where the function $H(x, y)$ is defined by (9). Substituting into (14) and changing the order of summing (its correctness is implied by absolute convergence), we obtain

$$
\begin{aligned}
\mathcal{F}\left(t ; z_{1}, z_{2} ; s_{1}, s_{2}\right) & \\
= & \sum_{\alpha_{1}, \alpha_{2}=0}^{\infty} \frac{z_{1}^{\alpha_{1}} z_{2}^{\alpha_{2}}}{\alpha_{1} ! \alpha_{2} !} \mathrm{e}^{z_{1} \mu /\left(\alpha_{2}+\mu\right)+z_{2}}\left(s_{1}-\frac{\mu}{\alpha_{2}+\mu}\right)^{\alpha_{1}}\left(s_{2}-1\right)^{\alpha_{2}} \mathrm{e}^{-\mu \alpha_{1} t} \\
& \times \int_{0}^{\infty} \int_{0}^{\infty} \mathrm{e}^{-\alpha_{1} t x-\alpha_{2} y} H(x, y) \mathrm{d} x \mathrm{~d} y \\
= & \int_{0}^{\infty} \int_{0}^{\infty} \mathrm{e}^{z_{2}}\left\{\sum_{\alpha_{2}=0}^{\infty} \frac{z_{2}^{\alpha_{2}}}{\alpha_{2} !} \mathrm{e}^{z_{1} \mu /\left(\alpha_{2}+\mu\right)}\left[\left(s_{2}-1\right) \mathrm{e}^{-y}\right]^{\alpha_{2}}\right. \\
= & \left.\int_{0}^{\infty} \int_{0}^{\infty} \mathrm{e}^{z_{1} s_{1} \mathrm{e}^{-(x+\mu) t}+z_{2}} \frac{z_{1}^{\alpha_{1}}}{\alpha_{1} !}\left[\left(s_{1}-\frac{\mu}{\alpha_{2}+\mu}\right) \mathrm{e}^{-(x+\mu) t}\right]^{\alpha_{1}}\right\} H(x, y) \mathrm{d} x \mathrm{~d} y \\
& \times\left\{\sum_{\alpha_{2}=0}^{\infty} \frac{\left[z_{2}\left(s_{2}-1\right) \mathrm{e}^{-y}\right]^{\alpha_{2}}}{\alpha_{2} !} \mathrm{e}^{z_{1} \mu\left(1-\mathrm{e}^{-(x+\mu) t}\right) /\left(\alpha_{2}+\mu\right)}\right\} H(x, y) \mathrm{d} x \mathrm{~d} y .
\end{aligned}
$$

For summing the series in brackets we use the equation

$$
\sum_{\alpha=0}^{\infty} \frac{b^{\alpha}}{\alpha !(\alpha+\mu)^{k}}=\frac{1}{(k-1) !} \int_{0}^{\infty} v^{k-1} \mathrm{e}^{-\mu v+b \mathrm{e}^{-v}} \mathrm{~d} v, \quad k=1,2, \ldots,
$$

and the modified Bessel function

$$
I_{1}(z)=\sum_{k=0}^{\infty} \frac{(z / 2)^{2 k+1}}{k !(k+1) !}
$$

(substituting $\left.a=z_{1} \mu\left(1-\mathrm{e}^{-(x+\mu) t}\right), b=z_{2}\left(s_{2}-1\right) \mathrm{e}^{-y}\right)$, to obtain

$$
\begin{aligned}
\sum_{\alpha_{2}=0}^{\infty} \frac{b^{\alpha_{2}}}{\alpha_{2} !} \mathrm{e}^{a /\left(\alpha_{2}+\mu\right)} & =\sum_{\alpha_{2}=0}^{\infty} \frac{b^{\alpha_{2}}}{\alpha_{2} !} \sum_{k=0}^{\infty} \frac{a^{k}}{k !\left(\alpha_{2}+\mu\right)^{k}} \\
& =\sum_{k=0}^{\infty} \frac{a^{k}}{k !} \sum_{\alpha_{2}=0}^{\infty} \frac{b^{\alpha_{2}}}{\alpha_{2} !\left(\alpha_{2}+\mu\right)^{k}}
\end{aligned}
$$




$$
\begin{aligned}
& =\mathrm{e}^{b}+\sum_{k=1}^{\infty} \frac{a^{k}}{k !(k-1) !} \int_{0}^{\infty} v^{k-1} \mathrm{e}^{-\mu v+b \mathrm{e}^{-v}} \mathrm{~d} v \\
& =\mathrm{e}^{b}+\int_{0}^{\infty} \sqrt{\frac{a}{v}} I_{1}(2 \sqrt{a v}) \mathrm{e}^{-\mu v+b \mathrm{e}^{-v}} \mathrm{~d} v .
\end{aligned}
$$

Using the well-known representation for the Bessel function,

$$
I_{1}(z)=I_{-1}(z)=\frac{1}{2 \pi \mathrm{i}} \oint_{0+} \mathrm{e}^{(z / 2)(u+1 / u)} \mathrm{d} u
$$

it is easy to obtain

$$
\sqrt{\frac{a}{v}} I_{1}(2 \sqrt{a v})=\frac{1}{2 \pi \mathrm{i}} \oint_{0+} \mathrm{e}^{v u+a / u} \mathrm{~d} u
$$

Substituting (17) into (16) and then (16) into (15), we have, finally,

$$
\begin{aligned}
\mathcal{F}\left(t ; z_{1}, z_{2} ; s_{1}, s_{2}\right) & \\
= & \int_{0}^{\infty} \int_{0}^{\infty} \exp \left(z_{1} s_{1} \mathrm{e}^{-(x+\mu) t}+z_{2}\right) \\
& \quad \times\left(\exp \left(z_{2}\left(s_{2}-1\right) \mathrm{e}^{-y}\right)+\int_{0}^{\infty}\left(\frac { 1 } { 2 \pi \mathrm { i } } \oint _ { 0 + } \operatorname { e x p } \left(z_{1} \mu \frac{1-\mathrm{e}^{-(x+\mu) t}}{u}\right.\right.\right. \\
& \left.\left.\left.\quad+z_{2}\left(s_{2}-1\right) \mathrm{e}^{-y-v}+(u-\mu) v\right) \mathrm{~d} u\right) \mathrm{~d} v\right) H(x, y) \mathrm{d} x \mathrm{~d} y .
\end{aligned}
$$

Combining the definition of the double generating function (6), (18), and using an exponent that expands

$$
\mathrm{e}^{z_{1} s_{1}+z_{2} s_{2}}=\sum_{\alpha_{1}, \alpha_{2}=0}^{\infty} \frac{z_{1}^{\alpha_{1}} z_{2}^{\alpha_{2}}}{\alpha_{1} ! \alpha_{2} !} s_{1}^{\alpha_{1}} s_{2}^{\alpha_{2}}
$$

we obtain the integral representation (10). Theorem 1 is proved.

Remark 1. We reduce the series (14) to the form

$$
\begin{aligned}
& \mathcal{F}\left(t ; z_{1}, z_{2} ; s_{1}, s_{2}\right) \\
& =\sum_{\alpha_{2}=0}^{\infty} \frac{z_{2}^{\alpha_{2}}}{\alpha_{2} !} \mathrm{e}^{z_{1} \mu /\left(\alpha_{2}+\mu\right)+z_{2}}\left(s_{2}-1\right)^{\alpha_{2}} \sum_{\alpha_{1}=0}^{\infty} \frac{z_{1}^{\alpha_{1}}}{\alpha_{1} !}\left(s_{1}-\frac{\mu}{\alpha_{2}+\mu}\right)^{\alpha_{1}}\left(\mathrm{e}^{-\left(\alpha_{2}+\mu\right) t}\right)^{\alpha_{1}} \\
& =\sum_{\alpha_{2}=0}^{\infty} \frac{z_{2}^{\alpha_{2}}\left(s_{2}-1\right)^{\alpha_{2}}}{\alpha_{2} !} \sum_{n=0}^{\infty} \frac{z_{2}^{n}}{n !} \exp \left(z_{1}\left[\frac{\mu}{\alpha_{2}+\mu}+\left(\frac{s_{1}-\mu}{\alpha_{2}+\mu}\right) \mathrm{e}^{-\left(\alpha_{2}+\mu\right) t}\right]\right) \\
& =\sum_{\alpha_{2}=0}^{\infty} z_{2}^{\alpha_{2}} \sum_{k=0}^{\alpha_{2}} \frac{\left(s_{2}-1\right)^{k}}{k !\left(\alpha_{2}-k\right) !} \sum_{\alpha_{1}=0}^{\infty} \frac{z_{1}^{\alpha_{1}}}{\alpha_{1} !}\left(s_{1} \mathrm{e}^{-(k+\mu) t}+\frac{\mu}{k+\mu}\left(1-\mathrm{e}^{-(k+\mu) t}\right)\right)^{\alpha_{1}} .
\end{aligned}
$$

In the resulting series (19) and the series (6), equating the coefficients of the identical powers of $z_{1}^{\alpha_{1}} z_{2}^{\alpha_{2}}$, we obtain the representation (cf. [15])

$$
F_{\left(\alpha_{1}, \alpha_{2}\right)}\left(t ; s_{1}, s_{2}\right)=\sum_{k=0}^{\alpha_{2}} C_{\alpha_{2}}^{k}\left(s_{1} \mathrm{e}^{-(k+\mu) t}+\frac{\mu}{k+\mu}\left(1-\mathrm{e}^{-(k+\mu) t}\right)\right)^{\alpha_{1}}\left(s_{2}-1\right)^{k} .
$$


Remark 2. Wishing to obtain a closed solution to (3) by the method of separating variables, one can encounter a difficult problem if $p_{1}<1$. We can assume that the first and the second Kolmogorov equation for the generating function could be solved by it. The jumps of the Markov process $\left(\xi_{1}(t), \xi_{2}(t)\right)$ are aimed in one direction, i.e. our process is 'a death process' (see Figure 1, case (a)). Therefore, in the $p_{1}<1$ case the series (11) exists. Also, we know that the generating function $F_{\left(\alpha_{1}, \alpha_{2}\right)}\left(t ; s_{1}, s_{2}\right)$ can be obtained in the form of a finite series in the $p_{1}=0$ case [5].

\section{Some consequences}

The initial conditions $\alpha_{1}, \alpha_{2}$ and the variables $s_{1}, s_{2}$ are involved in the integral equation (10) for the function $F_{\left(\alpha_{1}, \alpha_{2}\right)}\left(t ; s_{1}, s_{2}\right)$ as a simple arithmetic expression. This provides us with the standard methods for obtaining the following corollaries.

The moments of the Markov process $\left(\xi_{1}(t), \xi_{2}(t)\right)$ can be found from [1] and [12]. Thus,

$$
\begin{aligned}
& A_{i}(t)=\mathbb{E} \xi_{i}(t)=\left.\frac{\partial F_{\left(\alpha_{1}, \alpha_{2}\right)}}{\partial s_{i}}\right|_{s_{1}=1, s_{2}=1}, \\
& B_{i}(t)=\mathbb{E} \xi_{i}(t)\left(\xi_{i}(t)-1\right)=\left.\frac{\partial^{2} F_{\left(\alpha_{1}, \alpha_{2}\right)}}{\partial s_{i}^{2}}\right|_{s_{1}=1, s_{2}=1}, \\
& D_{i}(t)=\mathbb{D} \xi_{i}(t)=B_{i}(t)+A_{i}(t)-A_{i}^{2}(t), \quad i=1,2,
\end{aligned}
$$

where $\mathbb{E}$ is the expectation and $\mathbb{D}$ is the variance.

Corollary 1. For a Weiss epidemic process the expectations and variances are as follows:

$$
\begin{aligned}
A_{1}(t)= & \alpha_{1} \mathrm{e}^{-\mu t}, \quad A_{2}(t)=\alpha_{2}\left(\frac{\mu}{\mu+1}+\frac{1}{\mu+1} \mathrm{e}^{-(\mu+1) t}\right)^{\alpha_{1}} ; \\
D_{1}(t)= & \alpha_{1}\left(\mathrm{e}^{-\mu t}-\mathrm{e}^{-2 \mu t}\right) \\
D_{2}(t)= & \alpha_{2}\left(\alpha_{2}-1\right)\left(\frac{\mu}{\mu+2}+\frac{2}{\mu+2} \mathrm{e}^{-(\mu+2) t}\right)^{\alpha_{1}}+\alpha_{2}\left(\frac{\mu}{\mu+1}+\frac{1}{\mu+1} \mathrm{e}^{-(\mu+1) t}\right)^{\alpha_{1}} \\
& -\alpha_{2}^{2}\left(\frac{\mu}{\mu+1}+\frac{1}{\mu+1} \mathrm{e}^{-(\mu+1) t}\right)^{2 \alpha_{1}} .
\end{aligned}
$$

Proof. Let us calculate $A_{2}(t)$. Let

$$
\begin{aligned}
& A_{2}(t)=\left.\frac{\partial F_{\left(\alpha_{1}, \alpha_{2}\right)}}{\partial s_{2}}\right|_{s_{1}=1, s_{2}=1} \\
&=\int_{0}^{\infty} \int_{0}^{\infty}\left(\mathrm{e}^{-\alpha_{1}(x+\mu) t} \alpha_{2} \mathrm{e}^{-y}\right. \\
& \quad+\int_{0}^{\infty}\left(\frac{1}{2 \pi \mathrm{i}} \oint_{0+}\left(\frac{\mu}{u}\left(1-\mathrm{e}^{-(x+\mu) t}\right)+\mathrm{e}^{-(x+\mu) t}\right)^{\alpha_{1}}\right. \\
&\left.\left.\quad \times \alpha_{2} \mathrm{e}^{-y-v} \mathrm{e}^{(u-\mu) v} \mathrm{~d} u\right) \mathrm{~d} v\right) H(x, y) \mathrm{d} x \mathrm{~d} y
\end{aligned}
$$




$$
\begin{aligned}
& =\alpha_{2} \mathrm{e}^{-\alpha_{1}(\mu+1) t} \\
& +\alpha_{2} \int_{0}^{\infty} \int_{0}^{\infty} \int_{0}^{\infty}\left(\frac{1}{2 \pi \mathrm{i}} \oint_{0+} \sum_{k=0}^{\alpha_{1}} C_{\alpha_{1}}^{k} \frac{\mu^{k}}{u^{k}}\left(1-\mathrm{e}^{-(x+\mu) t}\right)^{k}\right. \\
& \left.\times\left(\mathrm{e}^{-(x+\mu) t}\right)^{\alpha_{1}-k} \mathrm{e}^{u v-(\mu+1) v-y} \mathrm{~d} u\right) H(x, y) \mathrm{d} v \mathrm{~d} x \mathrm{~d} y \\
& =\alpha_{2} \mathrm{e}^{-\alpha_{1}(\mu+1) t} \\
& +\alpha_{2} \int_{0}^{\infty} \int_{0}^{\infty} \int_{0}^{\infty} \sum_{k=1}^{\alpha_{1}} C_{\alpha_{1}}^{k} \frac{\mu^{k} v^{k-1}}{(k-1) !}\left(1-\mathrm{e}^{-(x+\mu) t}\right)^{k} \\
& \times\left(\mathrm{e}^{-(x+\mu) t}\right)^{\alpha_{1}-k} \mathrm{e}^{-(\mu+1) v-y} H(x, y) \mathrm{d} v \mathrm{~d} x \mathrm{~d} y \\
& =\alpha_{2} \mathrm{e}^{-\alpha_{1}(\mu+1) t} \\
& +\alpha_{2} \int_{0}^{\infty} \int_{0}^{\infty} \sum_{k=1}^{\alpha_{1}} C_{\alpha_{1}}^{k}\left(\frac{\mu}{\mu+1}\right)^{k} \sum_{l=0}^{k} C_{k}^{l}(-1)^{l}\left(\mathrm{e}^{-(x+\mu) t}\right)^{l} \\
& \times\left(\mathrm{e}^{-(x+\mu) t}\right)^{\alpha_{1}-k} \mathrm{e}^{-y} H(x, y) \mathrm{d} x \mathrm{~d} y \\
& =\alpha_{2} \mathrm{e}^{-\alpha_{1}(\mu+1) t}+\alpha_{2} \sum_{k=1}^{\alpha_{1}} C_{\alpha_{1}}^{k}\left(\frac{\mu}{\mu+1}\right)^{k} \sum_{l=0}^{k} C_{k}^{l}(-1)^{l} \mathrm{e}^{-l \mu t} \mathrm{e}^{-\left(\alpha_{1}-k\right) \mu t} \mathrm{e}^{-\left(l+\alpha_{1}-k\right) t} \\
& =\alpha_{2}\left(\mathrm{e}^{-(\mu+1) t}\right)^{\alpha_{1}}+\alpha_{2} \sum_{k=1}^{\alpha_{1}} C_{\alpha_{1}}^{k}\left(\frac{\mu}{\mu+1}\right)^{k}\left(1-\mathrm{e}^{-(\mu+1) t}\right)^{k}\left(\mathrm{e}^{-(\mu+1) t}\right)^{\alpha_{1}-k} \\
& =\alpha_{2}\left(\frac{\mu}{\mu+1}+\frac{1}{\mu+1} \mathrm{e}^{-(\mu+1) t}\right)^{\alpha_{1}} \text {. }
\end{aligned}
$$

We can calculate $A_{1}(t), B_{1}(t)$, and $B_{2}(t)$ in the same way. Corollary 1 is proved.

The states $\left\{\left(0, \gamma_{2}\right), \gamma_{2}=0,1,2, \ldots\right\}$ for the Markov process $\xi(t)$ are absorbing. For the final probabilities

$$
q_{\left(0, \gamma_{2}\right)}^{\left(\alpha_{1}, \alpha_{2}\right)}=\lim _{t \rightarrow \infty} P_{\left(0, \gamma_{2}\right)}^{\left(\alpha_{1}, \alpha_{2}\right)}(t)
$$

We introduce the generating function

$$
\Phi_{\left(\alpha_{1}, \alpha_{2}\right)}\left(s_{2}\right)=\sum_{\gamma_{2}=0}^{\infty} q_{\left(0, \gamma_{2}\right)}^{\left(\alpha_{1}, \alpha_{2}\right)} s_{2}^{\gamma_{2}}=\lim _{t \rightarrow \infty} F_{\left(\alpha_{1}, \alpha_{2}\right)}\left(t ; s_{1}, s_{2}\right) .
$$

Passing to the limit in (10) we obtain the integral expression which was obtained in [7] by direct solution of the stationary first Kolmogorov equation.

Corollary 2. The generating function of the final probabilities is as follows, $\mu>0, \alpha_{1}=$ $1,2, \ldots$,

$$
\Phi_{\left(\alpha_{1}, \alpha_{2}\right)}\left(s_{2}\right)=\frac{\mu^{\alpha_{1}}}{\left(\alpha_{1}-1\right) !} \int_{0}^{\infty} v^{\alpha_{1}-1}\left(1-\mathrm{e}^{-v}+s_{2} \mathrm{e}^{-v}\right)^{\alpha_{2}} \mathrm{e}^{-\mu v} \mathrm{~d} v
$$


Let us assume that at the initial moment the number, $\alpha_{1}$, of infected individuals is small and the number, $\alpha_{2}$, of susceptible individuals is large, which is the most interesting case for applications [2]. Using the explicit expression (10) for the probability distribution on $N^{2}$, and applying the characteristic functions method in a standard way (see [1] and [12]), we obtain the following corollary.

Corollary 3. Let us denote $\xi_{2}(t)$ as a number of particles of type $T_{2}$ at the moment $t$ for the Weiss epidemic process. We assume that there was $\alpha_{1}$ particles of type $T_{1}$ and $\alpha_{2}$ particles of type $T_{2}, \mu>0, \alpha_{1}=1,2, \ldots$, at the moment $t=0$. Then, for the fixed $t>0$, we have

$$
\lim _{\alpha_{2} \rightarrow \infty} \mathbb{P}\left\{\frac{\xi_{2}(t)}{\alpha_{2}} \leq x\right\}=F_{\alpha_{1}}(t ; x)
$$

where $F_{\alpha_{1}}(t ; x)$ is the distribution function, in which its characteristic function is equal to

$$
\begin{aligned}
\varphi_{\alpha_{1}}(t ; \lambda)= & \int_{-\infty}^{\infty} \mathrm{e}^{\mathrm{i} \lambda x} \mathrm{~d} F_{\alpha_{1}}(t ; x) \\
= & \mathrm{e}^{-\alpha_{1} \mu t} \mathrm{e}^{\mathrm{i} \lambda \mathrm{e}^{-\alpha_{1} t}}+\sum_{k=1}^{\alpha_{1}} C_{\alpha_{1}}^{k} \frac{\mu^{k}}{(k-1) !} \sum_{l=0}^{k} C_{k}^{l}(-1)^{l} \\
& \times \int_{0}^{\mathrm{e}^{-\left(\alpha_{1}-k+l\right) t}} \mathrm{e}^{\mathrm{i} \lambda x} x^{\mu-1}\left(-\ln x-\left(\alpha_{1}-k+l\right) t\right)^{k-1} \mathrm{~d} x .
\end{aligned}
$$

From (22) we can find the distribution function expression

$$
F_{\alpha_{1}}(t ; x)= \begin{cases}0, & x<\mathrm{e}^{-\alpha_{1} t} \\ \mathrm{e}^{-\alpha_{1} \mu t}+\int_{\mathrm{e}^{-\alpha_{1} t}}^{x} f_{\alpha_{1}}(t ; y) \mathrm{d} y, & \mathrm{e}^{-\alpha_{1} t} \leq x<1 \\ 1, & 1 \leq x,\end{cases}
$$

where the piecewise-continuous function $f_{\alpha_{1}}(t ; x)$ at each interval $\left(\mathrm{e}^{-\left(\alpha_{1}-n\right) t}, \mathrm{e}^{-\left(\alpha_{1}-n-1\right) t}\right)$, $n=0, \ldots, \alpha_{1}-1$, is defined as

$$
f_{\alpha_{1}}(t ; x)=x^{\mu-1} \sum_{k=n+1}^{\alpha_{1}} C_{\alpha_{1}}^{k} \frac{\mu^{k}}{(k-1) !} \sum_{l=0}^{k-n-1} C_{k}^{l}(-1)^{l}\left(-\ln x-\left(\alpha_{1}-k+l\right) t\right)^{k-1} .
$$

In particular,

$$
\begin{gathered}
F_{1}(t ; x)= \begin{cases}0, & x<\mathrm{e}^{-t} \\
x^{\mu}, & \mathrm{e}^{-t} \leq x<1 \\
1, & 1 \leq x,\end{cases} \\
F_{2}(t ; x)= \begin{cases}0, & x<\mathrm{e}^{-2 t} \\
x^{\mu}(1+\mu \ln x+2 \mu t), & \mathrm{e}^{-2 t} \leq x<\mathrm{e}^{-t} \\
x^{\mu}(1-\mu \ln x), & \mathrm{e}^{-t} \leq x<1 ; \\
1, & 1 \leq x .\end{cases}
\end{gathered}
$$

Note that deducing the limit theorem (21) from the representation (20) is challenging, but deducing (21) from (10) is simple and consists of using the $\operatorname{limit}_{n \rightarrow \infty}(1+1 / n)^{n}=\mathrm{e}$. 
The main result of this paper is the limit theorem (21). It is the statement of a 'threshold theorem' type [2]. As stated above the asymptotic properties of the component $\xi_{2}(t)$ of the process as $\alpha_{2} \rightarrow \infty$ are interesting for practical use. Theorems of such kind are used to check the threshold number of infected individuals and when this number has been exceeded it means that the epidemic has begun.

\section{References}

[1] Athreya, K. B. And Ney, P. E. (1972). Branching Processes. Springer, New York.

[2] Bailey, N. T. J. (1975). The Mathematical Theory of Infectious Diseases and Its Applications, 2nd edn. Hafner, New York.

[3] Bartlett, M. S. (1955). An Introduction to Stochastic Processes, With Special Reference to Methods and Applications. Cambridge University Press.

[4] Ditkin, V. A. And Prudnikov, A. P. (1962). Operational Calculus in Two Variables and Its Applications. Pergamon, New York.

[5] Gani, J. (1965). On a partial differential equation of epidemic theory. I. Biometrika 52, 617-622.

[6] Hazewinkel, M. (ed.) (1989). Epidemic process. In Encyclopaedia of Mathematics, Vol. 3, Kluwer, Dordrecht.

[7] Kalinkin, A. V. (1999). Final probabilities for a branching process with interaction of particles and an epidemic process. Theory Prob. Appl. 43, 633-640.

[8] Kalinkin, A. V. (2002). Markov branching processes with interaction. Russian Math. Surveys 57, $241-304$.

[9] Mastikhin, A. V. (2007). Final distribution for the Markov process of the Gani epidemic. Math. Notes 82, 787-797.

[10] Mastikhin, A. V. (2012). Final probabilities for Becker epidemic Markov process. Theory Prob. Appl. 56, 521-527.

[11] Sakino, S. (1968). On the solution of the epidemic equations. Ann. Inst. Statist. Math. Suppl. 5, 9-19.

[12] Sevast’yanov, B. A. (1971). Vetvyashchiesya Protsessy (Branching Processes). Nauka, Moscow. (In Russian.) (Also available in German: Sewastuanow, B. A. (1974). Verzweigungsprozesse. Akademie-Verlag, Berlin.)

[13] Sevast'yanov, B. A. And Kalinkin, A. V. (1982). Random branching processes with interaction of particles. Soviet Math. Dokl. 25, 644-646.

[14] Siskind, V. (1965). A solution of the general stochastic epidemic. Biometrics 52, 613-616.

[15] WeIss, G. H. (1965). On the spread of epidemics by carriers. Biometrics 21, 481-490. 